\title{
Myocardial Stunning After Electrocution With Complete Reversibility Within 24 Hours: Role of Repeat Transthoracic Echocardiograms in Potential Cardiac Transplant Donors
}

\author{
Hassan Tahir ${ }^{\mathrm{a}, \mathrm{c}}$, Sarina Sachdev ${ }^{\mathrm{a}}$, Landai Nguyen ${ }^{\mathrm{a}}$, Nikky Bardia ${ }^{\mathrm{a}}$, \\ Bassam Omar ${ }^{\mathrm{a}}$, Michael V. Cohen ${ }^{\mathrm{a}, \mathrm{b}}$
}

\begin{abstract}
Despite the development of ventricular assist devices, cardiac transplantation remains an important procedure for patients with advanced heart failure. The number of transplants done annually has remained stable because of lack of of donors. Left ventricular systolic dysfunction remains one of the most important reasons for seeking a donor heart. Myocardial stunning is an important cause of reversible systolic dysfunction. Electrical injury is a recognized cause of myocardial stunning with variable duration ranging from days to weeks. Repeating the transthoracic echocardiogram to look for reversibility of left ventricular dysfunction can be a cost-effective method to improve the selection of heart donors. This can significantly help to decrease critical organ shortage. We present a case of myocardial stunning after electrocution which was completely reversible within a few hours, thus meeting cardiac transplant donor criteria.
\end{abstract}

Keywords: Cardiac transplant donor; Electrocution; Myocardial stunning; Serial echocardiography

\section{Introduction}

Cardiac transplantation is an important procedure which is known to improve morbidity and mortality in patients with advanced heart failure [1]. One of the most important criteria used to select the donor is normal left ventricular systolic function [2]. Myocardial stunning is a reversible form of systolic dysfunction which typically occurs in response to transient myocardial ischemia. But any insult to the myocardium

Manuscript submitted June 28, 2018, accepted July 23, 2018

aDivision of Cardiology, Department of Medicine, University of South Alabama, Mobile, AL, USA

bDepartment of Physiology and Cell Biology, University of South Alabama, Mobile, AL, USA

${ }^{\mathrm{c} C o r r e s p o n d i n g ~ A u t h o r: ~ H a s s a n ~ T a h i r, ~ D i v i s i o n ~ o f ~ C a r d i o l o g y, ~ D e p a r t m e n t ~ o f ~}$ Medicine, University of South Alabama, 2451 USA Medical Center Dr, Room 10B, Mobile, AL 36617, USA. Email: htahir@health.southalabama.edu

doi: https://doi.org/10.14740/cr754w can lead to myocardial stunning which can last from days to weeks and which depends on the duration of the preceding ischemic event or other insult. For example, brief episodes of myocardial stunning have been observed after prolonged emotional trauma resulting in the well described takotsubo cardiomyopathy [2]. We present a case of myocardial stunning after electrocution which was completely reversible within a few hours thus enabling use of the heart for transplantation.

\section{Case Report}

A 32-year-old Caucasian male with no past medical history sustained an electrical injury at work and was found to be in cardiac arrest. He underwent 20 min of CPR with spontaneous return of circulation. He was intubated in the field and was admitted to an intensive care unit. On examination, he had a large electrical burn on the left anterior chest and an exit wound in the left hand. Cardiovascular examination revealed normal heart sounds with no murmur. The EKG showed sinus tachycardia with no ST or T-wave changes suggestive of ischemia (Fig. 1). Laboratory investigations were unremarkable except for significantly elevated cardiac markers (troponin $1.14 \mathrm{ng}$ / $\mathrm{mL}$ and CK MB $402 \mathrm{ng} / \mathrm{mL}$ ). CT scans of head, cervical spine, chest, abdomen and pelvis did not show any acute pathology. CXR showed mild pulmonary congestion (Fig. 2). A transthoracic echocardiogram showed severely depressed left ventricular systolic function with an ejection fraction of $20 \%$ (Fig. 3 ). His condition deteriorated and the patient had recurrent seizures. Repeat CT scan of the head showed diffuse cerebral edema with impending herniation. He did not have brainstem reflexes on examination. He was diagnosed with anoxic brain injury and was declared brain-dead. He was evaluated by the transplant team for multiple organ donations. Initially it was felt that the left ventricular dysfunction precluded harvesting of the heart. A decision was made to repeat the transthoracic echocardiogram $24 \mathrm{~h}$ after admission. To our surprise, his left ventricular systolic function had completely normalized with an ejection fraction of 55-60\% (Fig. 4). He underwent coronary angiography to evaluate his coronary anatomy which was normal (Fig. 5). After thorough evaluation, he was considered to be an ideal cardiac donor. 


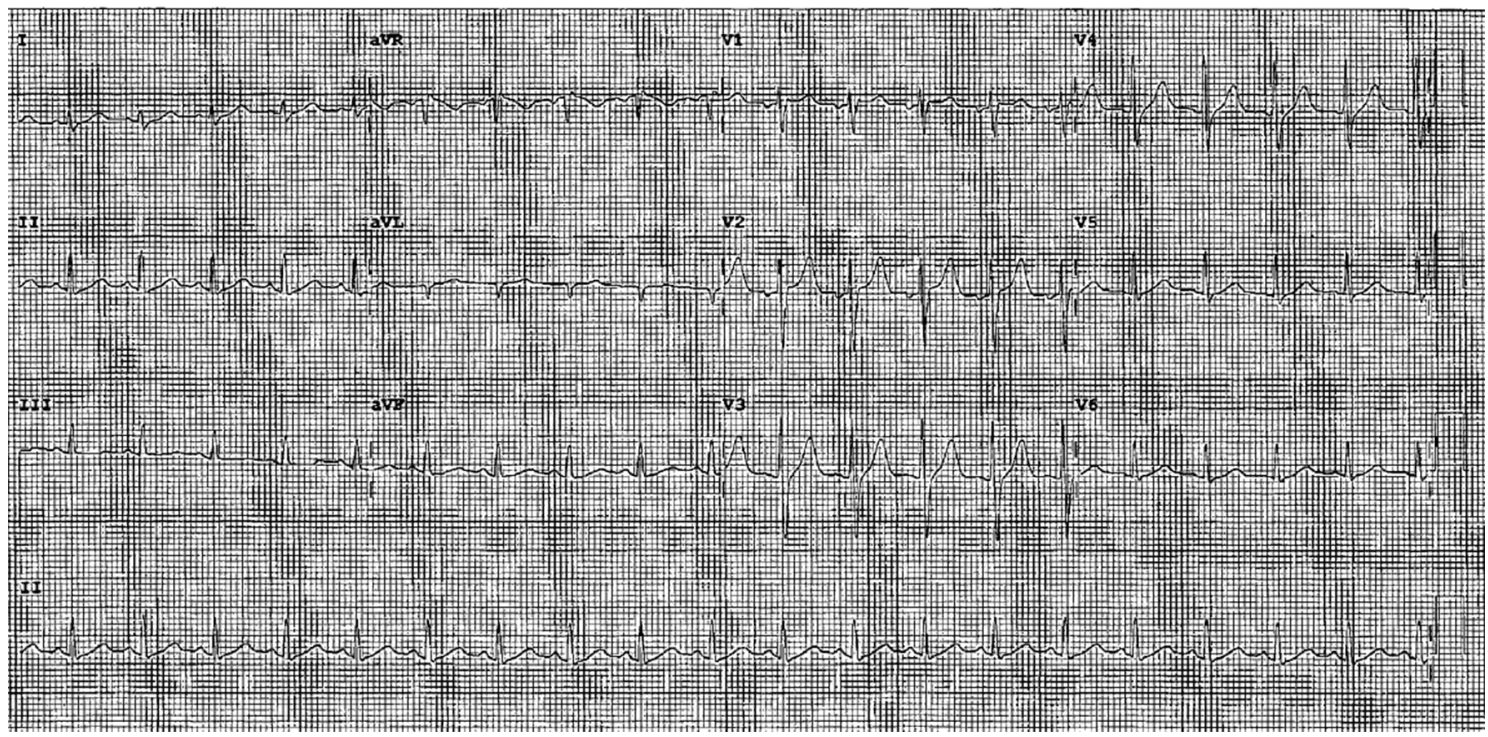

Figure 1. EKG showed sinus tachycardia with no ST or T-wave changes suggestive of ischemia.

\section{Discussion}

Cardiac transplantation for advanced heart failure is an important treatment option which can significantly improve quality of life in appropriately selected patients. Adequate preoperative evaluation, improvement in surgical techniques and newer immunosuppressive agents have significantly improved survival rates [1]. Almost 2,000 cardiac transplants are performed annually in the USA [1]. However, the number of cardiac transplants in the USA has remained steady in the last decade because of absence of suitable donors. This critical organ shortage underscores the importance of adequate donor selection by healthcare professionals. Traditional criteria used to select potential donors include age less than 55 years, no history of chest trauma or cardiac disease, no prolonged hypotension or hypoxemia, appropriate hemodynamics, normal electrocardiogram, normal

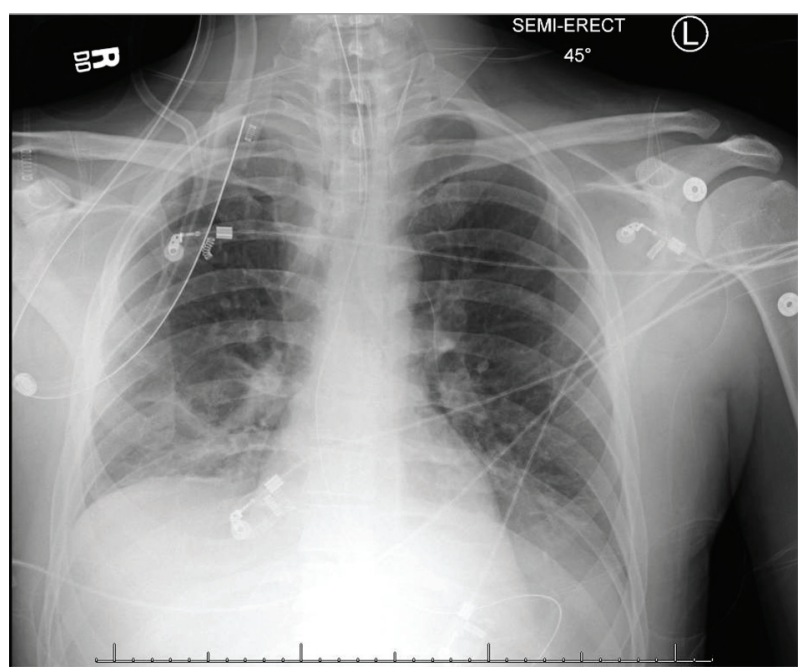

Figure 2. CXR showed mild pulmonary congestion. echocardiogram, normal coronary angiography and negative hepatitis and HIV serology [3]. Expanded donor criteria were published in 2001 to increase the selection of donor hearts and decrease the increasing gap between the number of heart donors and recipients [4]. Out of many reasons for non-selection of a donor heart, cardiac dysfunction is the most common cause which probably accounts for up to $26 \%$ of donor rejections [5].

Myocardial stunning is a reversible reduction in the contractile function of the heart. Initially stunning was felt to be related to a reversible episode of myocardial ischemia [6]. This phenomenon is different from myocardial hibernation where contractile function is depressed for a prolonged time because of chronic ischemia [6]. Any insult leading to transient myocardial ischemia can cause myocardial stunning such as coronary angioplasty, thrombolysis, acute coronary syndrome and coronary artery bypass graft surgery [6]. More recently, myocardial stunning has also been associated with conditions characterized by high catecholamine levels such as stroke, subarachnoid hemorrhage and intense emotions [7]. Myocardial stunning can be seen in up to $40 \%$ of patients with brain death [8]. It has also been reported after successful cardioversion of atrial fibrillation [9] and electrocution.

Electrical injuries are life-threatening occupational health hazards which can cause sudden cardiac arrest by inducing cardiac dysrhythmias. Ventricular fibrillation is considered the main cause of death in patients with electrical injury [10]. Other cardiac effects include myocardial necrosis, hemorrhagic pericarditis and coronary vasospasm resulting in myocardial infarction [11]. Table 1 [10-15] describes the various cardiac effects of electrical injury reported in the literature. Electrical injury can also lead to myocardial stunning in the absence of reversible myocardial injury, the exact mechanism of which is unknown [11]. Systolic dysfunction is completely reversible in most of the cases [12]. The duration of systolic dysfunction can vary from days to weeks. Table 2 [15-19] shows the duration of myocardial stunning from various case studies in patients with 


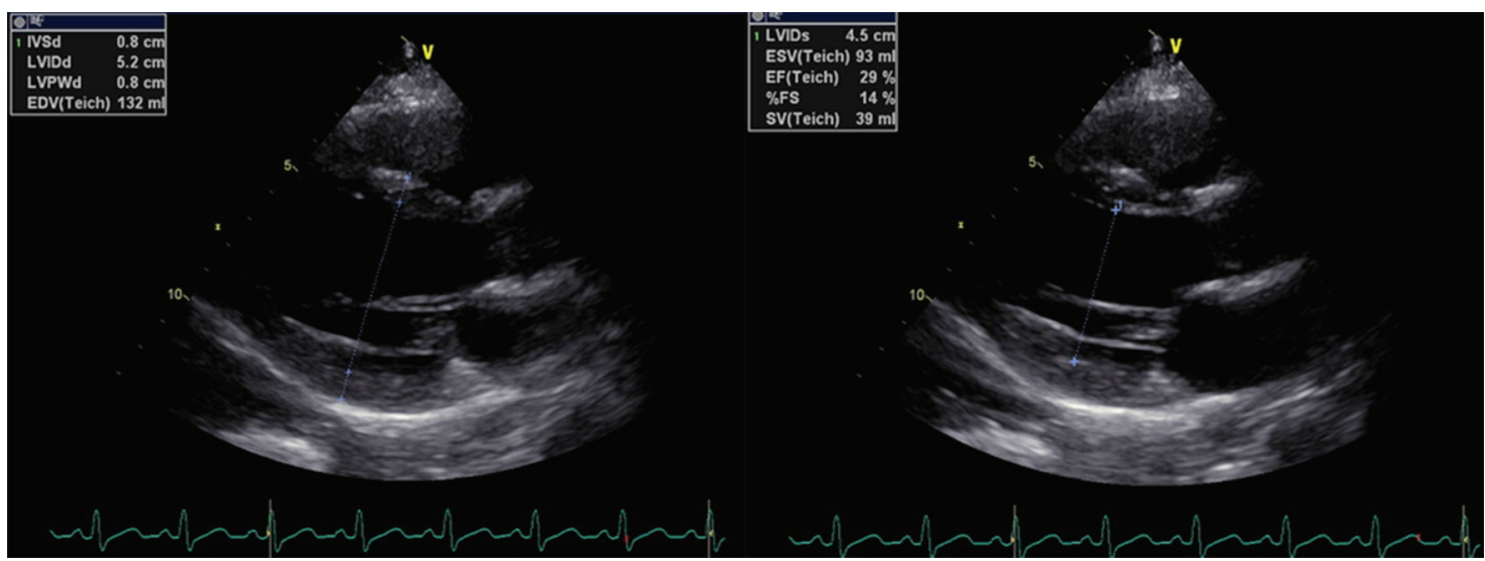

Figure 3. Transthoracic echocardiogram showing severely depressed left ventricular systolic function with an ejection fraction of $20 \%$.

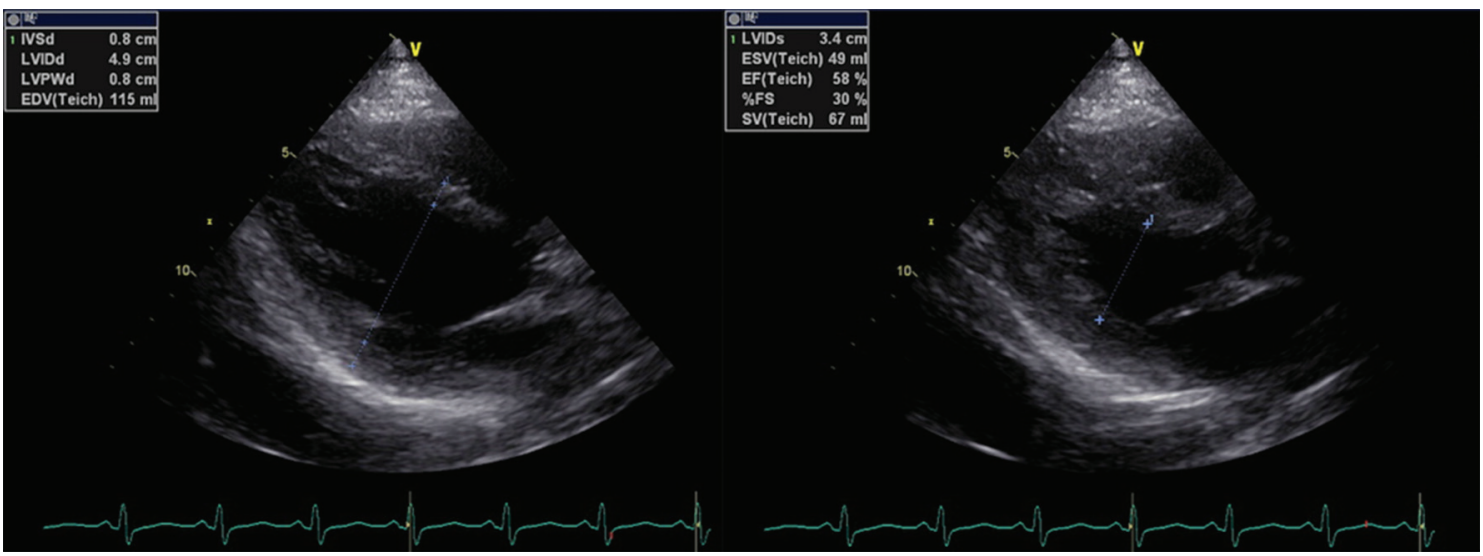

Figure 4. Transthoracic echocardiogram done after $24 \mathrm{~h}$ showing completely normal left ventricular systolic function with an ejection fraction of $55-60 \%$.

different forms of electrical accidents. As indicated in Table 2, the duration of myocardial stunning is highly variable which may be dependent on the type of electrical injury, the voltage of the insult and the degree of potential ischemia $[10,11]$.

As occupational electrical injuries most commonly occur in young healthy workers, it is important to screen these pa-

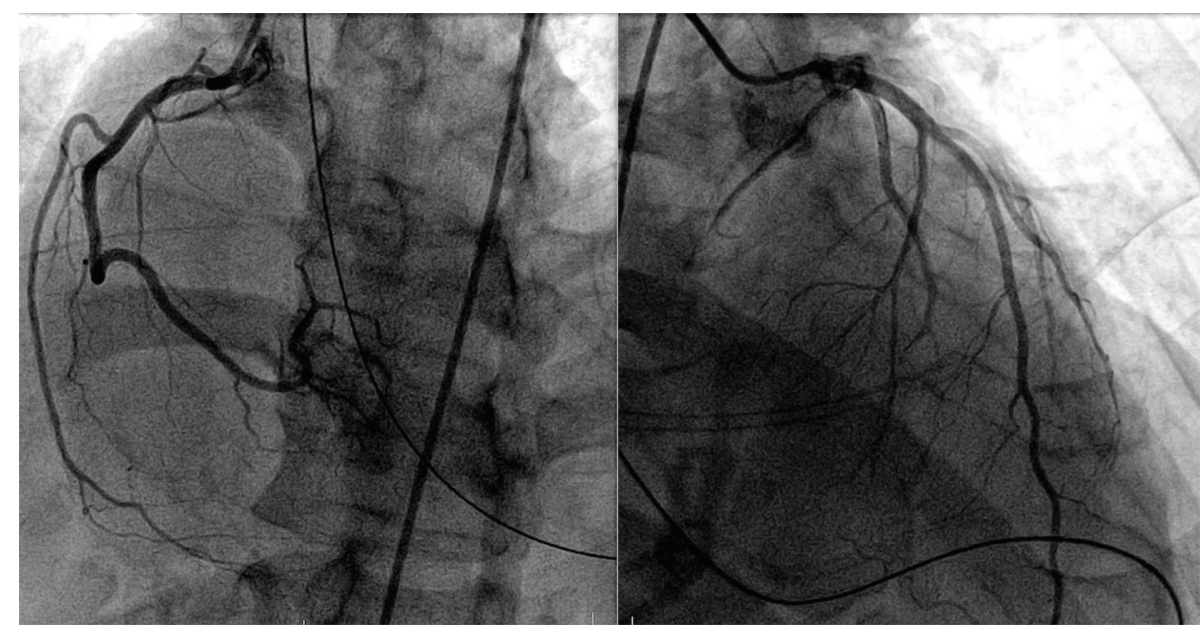

Figure 5. Coronary angiography evaluating patient's coronary anatomy which was normal. 
Table 1. Cardiac Effects of Electrical Injury [10-15]

\begin{tabular}{l}
\hline Arrhythmias [10] \\
Myocardial infarction [11] \\
Myocardial ischemia without necrosis [11] \\
Coronary vasospasm [11] \\
Acute heart failure [10,11] \\
Cardiogenic shock [10,11] \\
Non-specific EKG changes without cardiac pathology [10-12] \\
Complete atrioventricular block [13] \\
Symptomatic bradycardia [14] \\
Takotsubo cardiomyopathy [15]
\end{tabular}

tients for heart donation if there is brain death. It is reasonable to repeat the transthoracic echocardiogram within $24-72$ $\mathrm{h}$ in such patients if their initial echocardiogram shows left ventricular systolic dysfunction. Repeating the transthoracic echocardiogram can be a cost-effective strategy to identify patients with reversible myocardial dysfunction who may be potential candidates for donation. Recently, the effect of pharmacological stress echocardiography in potential, brain-dead donors with systolic dysfunction was studied by Bombardini et al [20]. Their study showed promising results when donor hearts with improved systolic function on the stress echocardiogram were transplanted. All three patients studied by Bombardini et al had no allograft rejection and normal LV systolic function 1 month after transplant and all were alive at 1 year [20]. Our patient had cardiac arrest with brain death after electrical injury. His initial transthoracic echocardiogram showed severe systolic dysfunction which was completely reversible as shown by the repeat echocardiogram after $24 \mathrm{~h}$. A single echocardiographic assessment is inadequate to predict reversibility of systolic dysfunction. A donor heart should not be rejected based on the initial ejection fraction especially in young brain-dead patients with electrical injury.

\section{Conclusion}

Left ventricular dysfunction is one of the most important reasons for donor heart rejection. Myocardial stunning is a reversible form of myocardial dysfunction that occurs after reversible ischemia or catecholamine surge. The duration of myocardial stunning is variable. However, recovery might be quick in some cases like electrocution, especially in young previously healthy patients. It is reasonable to repeat the transthoracic echocardiogram in such patients which will likely help to increase the number of heart donors. Further studies are needed to examine the effect of electrical injury on duration and severity of myocardial stunning.

\section{Conflict of Interest}

None.
Table 2. Duration of Myocardial Stunning After Electrical Injuries [15-19]

\begin{tabular}{ll}
\hline Case study & Duration of myocardial stunning \\
\hline Hyashi et al [16] & 2 days \\
Dundon et al [15] & 6 weeks \\
Rivera et al [17] & 9 days \\
McGill et al [18] & 3 months \\
Lewin et al [19] & 3 days \\
\hline
\end{tabular}

\section{Funding}

None.

\section{References}

1. Everly MJ. Cardiac transplantation in the United States: an analysis of the UNOS registry. Clin Transpl. 2008:3543.

2. Kilic A, Emani S, Sai-Sudhakar CB, Higgins RS, Whitson BA. Donor selection in heart transplantation. J Thorac Dis. 2014;6(8):1097-1104.

3. John R. Donor management and selection for heart transplantation. Semin Thorac Cardiovasc Surg. 2004;16(4):364-369.

4. Zaroff JG, Rosengard BR, Armstrong WF, Babcock WD, D'Alessandro A, Dec GW, Edwards NM, et al. Consensus conference report: maximizing use of organs recovered from the cadaver donor: cardiac recommendations, March 28-29, 2001, Crystal City, Va. Circulation. 2002;106(7):836-841.

5. Zaroff JG, Babcock WD, Shiboski SC. The impact of left ventricular dysfunction on cardiac donor transplant rates. J Heart Lung Transplant. 2003;22(3):334-337.

6. Camici PG, Prasad SK, Rimoldi OE. Stunning, hibernation, and assessment of myocardial viability. Circulation. 2008;117(1):103-114.

7. Kloner RA, Arimie RB, Kay GL, Cannom D, Matthews $\mathrm{R}$, Bhandari A, Shook T, et al. Evidence for stunned myocardium in humans: a 2001 update. Coron Artery Dis. 2001;12(5):349-356.

8. Dujardin KS, McCully RB, Wijdicks EF, Tazelaar HD, Seward JB, McGregor CG, Olson LJ. Myocardial dysfunction associated with brain death: clinical, echocardiographic, and pathologic features. J Heart Lung Transplant. 2001;20(3):350-357.

9. Sandroni C, Sanna T, Cavallaro F, Caricato A. Myocardial stunning after successful defibrillation. Resuscitation. 2008;76(1):3-4.

10. Koumbourlis AC. Electrical injuries. Crit Care Med. 2002;30(11 Suppl):S424-430.

11. Ku CS, Lin SL, Hsu TL, Wang SP, Chang MS. Myocardial damage associated with electrical injury. Am Heart J. 1989;118(3):621-624.

12. Lichtenberg R, Dries D, Ward K, Marshall W, Scanlon 
P. Cardiovascular effects of lightning strikes. J Am Coll Cardiol. 1993;21(2):531-536.

13. Osman B, Tolga HE, Hakki K, Murat B, Lale DA, Mehmet BY. Electrical Injury-induced complete atrioventricular block: is permanent pacemaker required. Case Rep Cardiol. 2015;2:1-3.

14. Yew KL. Electrocution induced symptomatic bradycardia necessitating pacemaker implantation. Heart Views. 2014;15(2):49-50.

15. Dundon BK, Puri R, Leong DP, Worthley MI. Takotsubo cardiomyopathy following lightning strike. Emerg Med J. 2008;25(7):460-461.

16. Hayashi M, Yamada H, Agatsuma T, Nomura H, Kitahara O. A case of takotsubo-shaped hypokinesis of the left ventricle caused by a lightning strike. Int Heart J.
2005;46(5):933-938.

17. Rivera J, Romero KA, Gonzalez-Chon O, Uruchurtu E, Marquez MF, Guevara M. Severe stunned myocardium after lightning strike. Crit Care Med. 2007;35(1):280285.

18. McGill MP, Kamp TJ, Rahko PS. High-voltage injury resulting in permanent right heart dysfunction. Chest. 1999;115(2):586-587.

19. Lewin RF, Arditti A, Sclarovsky S. Non-invasive evaluation of electrical cardiac injury. $\mathrm{Br}$ Heart J. 1983;49(2):190-192.

20. Bombardini T, Gherardi S, Leone O, Sicari R, Picano E. Transplant of stunned donor hearts rescued by pharmacological stress echocardiography: a "proof of concept" report. Cardiovasc Ultrasound. 2013;11:27. 